KUNS-1612

hep-th/9910196

\title{
Brane Configuration from Monopole Solution in Non-Commutative Super Yang-Mills Theory
}

\author{
Koji Hashimoto, Hiroyuki Hata ${ }^{\dagger}$ and Sanefumi Moriyama \\ Department of Physics, Kyoto University, Kyoto 606-8502, Japan
}

October, 1999

\begin{abstract}
We study the structure of the monopole configuration in $U(2)$ non-commutative super Yang-Mills theory. Our analysis consists of two steps: solving the BPS equation and then the eigenvalue equation in the non-commutative space. Calculation to the first non-trivial order in the non-commutativity parameter $\theta$ shows that the monopole exhibits a certain non-locality. This structure is precisely the one expected from the recent predictions by the brane-configuration technique.
\end{abstract}

*hasshan@gauge.scphys.kyoto-u.ac.jp

thata@gauge.scphys.kyoto-u.ac.jp

${ }_{\ddagger}^{\ddagger}$ moriyama@gauge.scphys.kyoto-u.ac.jp 


\section{Introduction}

The fertile developments in string theory in this half a decade have enabled us to understand various perturbative and non-perturbative phenomena in field theories by geometrical intuitive pictures. This progress in string theory is now beyond the province of reproduction of the result of the field theories, that is, now the string theory has predictive power in various field theories.

One of the most intriguing examples is the $1 / 4$ BPS dyon solution in 4-dimensional $\mathcal{N}=4$ super Yang-Mills theory (SYM). The study of the $1 / 4$ BPS states in SYM was triggered by the discovery of the stable string network in type IIB string theory $[1,2]$. When this string network has its ends on D3-branes, these states preserve $1 / 4$ supersymmetries of the original D3-brane worldvolume theory [3]. After the study from the string theory side, there appeared some works $[4,5,6,7]$ in which explicit field theoretical solutions for the corresponding solitons were constructed. The properties of the solution favor the string theory interpretation with respect to their $(p, q)$-charges, masses and supersymmetries.

Recent topics of the string-theoretical realization of field theories are concerning the space non-commutativity $[8,9,10]$. The non-commutative super Yang-Mills theory (NCSYM) is realized as a decoupling limit of the worldvolume theory on D3-branes in the non-trivial NS-NS 2-form background. Taking advantage of this equivalence, some basic properties of localized objects in this exotic field theory were analyzed [11]. The brane configurations corresponding to the monopoles, dyons and 1/4 BPS dyons were constructed, and they were shown to have the same masses and supersymmetry properties as the ordinary SYM counterparts. One fascinating prediction from this brane technique is that the monopole in the NCSYM has non-locality $\delta$ due to the tilt of the D-string suspended between two D3branes (see fig. 1). Believing that the brane configuration of this figure precisely captures the field theoretical properties, the configuration of the monopole in the NCSYM should reproduce the tilted line, as the eigenvalues of the Higgs field. The ends of the D-string appear to be magnetic charges, hence the field theoretical solution should contain dipole structure.

In this paper, we explicitly solve the BPS equation for the monopole of the NCSYM to the first non-trivial order in $\theta_{i j}$ which specifies the non-commutativity. This $1 / 2$ BPS solution has the same mass as the ordinary SYM monopole, in agreement with the prediction by string theory. Solving the non-commutative eigenvalue equation in Sec. 3, we show in Sec. 4 that the solution actually reproduces the tilt of the suspended D-string. Examining the magnetic field, the dipole structure is also found. In the final section, we summarize the paper and give some discussions. 


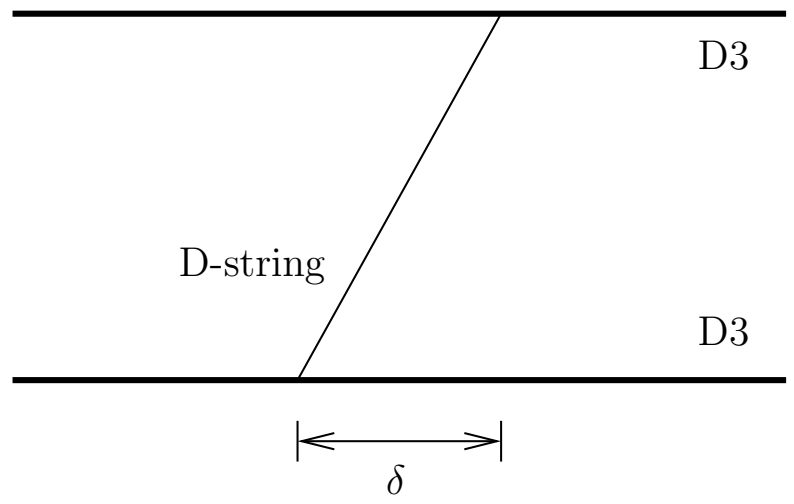

Figure 1: The D-string suspended between two parallel D3-branes tilts owing to the background of the constant NS-NS $B$-field.

\section{Non-commutative BPS equation and its solution}

The Bogomol'nyi-Prasad-Sommerfield (BPS) monopole solution $[12,13]$ of the ordinary SYM is saturating a particular energy bound which is usually called the BPS bound. Since this bound is topologically sensible, the state saturating the bound is stable. Now in the case of the NCSYM, unfortunately the topological argument seems not to be valid due to the high complexity of the $*$-product. Even with this complexity, we can argue a similar mass bound as in the following [11].

The energy of the system without the electric field is given by

$$
E=\operatorname{Tr} \int d^{3} x\left[\frac{1}{2} F_{i j} * F_{i j}+D_{i} \Phi * D_{i} \Phi\right],
$$

where we have defined the field strength and the covariant derivative as

$$
\begin{aligned}
F_{i j} & \equiv \partial_{i} A_{j}-\partial_{j} A_{i}-i A_{i} * A_{j}+i A_{j} * A_{i}, \\
D_{i} \Phi & \equiv \partial_{i} \Phi-i A_{i} * \Phi+i \Phi * A_{i} .
\end{aligned}
$$

We have put the Yang-Mills coupling constant $g_{\mathrm{YM}}$ equal to unity. The $*$-product is defined with the non-commutativity parameter $\theta_{i j}$ by

$$
(f * g)(x) \equiv f(x) \exp \left(\frac{i}{2} \theta_{i j} \overleftarrow{\partial_{i}} \overrightarrow{\partial_{j}}\right) g(x)=f(x) g(x)+\frac{i}{2}\{f, g\}(x)+O\left(\theta^{2}\right)
$$

where $\{f, g\}$ is the Poisson bracket,

$$
\{f, g\}(x) \equiv \theta_{i j} \partial_{i} f(x) \partial_{j} g(x)
$$


We shall take as the gauge group the simplest one $U(2)$. Note that the group $S U(2)$ is not allowed here since the algebra of any special unitary group is not closed when the multiplication is defined by the *-product. The energy $(2.1)$ is bounded below by a surface integral as follows:*

$$
\begin{aligned}
E & =\frac{1}{2} \operatorname{Tr} \int d^{3} x\left[\mp \epsilon_{i j k}\left(F_{i j} * D_{k} \Phi+D_{k} \Phi * F_{i j}\right)+\left(F_{i j} \pm \epsilon_{i j k} D_{k} \Phi\right) *\left(F_{i j} \pm \epsilon_{i j l} D_{l} \Phi\right)\right] \\
& \geq \operatorname{Tr} \int d^{3} x \partial_{k}\left[\mp \epsilon_{i j k}\left(F_{i j} * \Phi\right)\right] .
\end{aligned}
$$

If the solution of the non-commutative BPS (NCBPS) equation,

$$
D_{i} \Phi+\frac{1}{2} \epsilon_{i j k} F_{j k}=0
$$

has the same asymptotic behavior as the ordinary BPS solution, then the energy remains the same. This fact will be confirmed to the first non-trivial order in $\theta$ by explicitly solving the NCBPS equation (2.7).

We shall solve the NCBPS equation $(2.7)$ to $O(\theta)$ in the small $\theta$ expansion. Let us express the gauge field as

$$
A_{i} \equiv\left(A_{i}^{a}+a_{i}^{a}\right) \frac{1}{2} \sigma_{a}+\left(A_{i}^{0}+a_{i}^{0}\right) \frac{1}{2} \mathbb{I},
$$

where the upper (lower) case component fields are of $O\left(\theta^{0}\right)\left(O\left(\theta^{1}\right)\right)$. The scalar $\Phi$ is expressed in a similar manner. First, the $O\left(\theta^{0}\right)$ part of the NCBPS equation (2.7) is nothing but the ordinary BPS equation, and we adopt the well-known BPS monopole solution as the $O\left(\theta^{0}\right)$ part of the solution:

$$
\Phi^{a}=\frac{x^{a}}{r} F(r), \quad A_{i}^{a}=\epsilon_{a i j} \frac{x_{j}}{r} W(r), \quad \Phi^{0}=A_{i}^{0}=0,
$$

where the the functions appearing in the solution are

$$
F(r) \equiv C \operatorname{coth}(C r)-\frac{1}{r}, \quad W(r) \equiv \frac{1}{r}-\frac{C}{\sinh (C r)} .
$$

The dimensionful parameter $C$ determines the mass scale of the monopole. For later convenience, we present the asymptotic behavior of these functions:

$$
F(r)=C-\frac{1}{r}+O\left(e^{-C r}\right), \quad W(r)=\frac{1}{r}+O\left(e^{-C r}\right) .
$$

Next let us proceed to the $O(\theta)$ part of the NCBPS equation (2.7). Plugging (2.8) into the NCBPS equation (2.7) and taking the $O(\theta)$ part, the $U(1)$ component reads

$$
\partial_{i} \varphi^{0}+\frac{1}{2}\left\{A_{i}^{a}, \Phi^{a}\right\}+\epsilon_{i j k}\left(\partial_{j} a_{k}^{0}+\frac{1}{4}\left\{A_{j}^{a}, A_{k}^{a}\right\}\right)=0
$$

${ }^{*}$ In deriving the inequality (2.6), we assumed that $F_{i j} \pm \epsilon_{i j k} D_{k} \Phi$ decay sufficiently fast at the infinity so that we can apply the formula $\int d^{3} x(f * g)(x)=\int d^{3} x f(x) g(x)$ for these quantities. 
and does not contain the $S U(2)$ fields $\left(a_{i}^{a}, \varphi^{a}\right)$. On the other hand, the $S U(2)$ component of the $O(\theta)$ part of the NCBPS equation decouples from the $U(1)$ fields, and is in fact the linearized equation for the fluctuation $\left(a_{i}^{a}, \varphi^{a}\right)$ obtained from the ordinary BPS equation. Since any solution for $\left(a_{i}^{a}, \varphi^{a}\right)$ corresponds to a $\theta$-dependent gauge transformation on the BPS solution (2.9), we take $a_{i}^{a}=\varphi^{a}=0$ hereafter.

Now our task is to solve the equation (2.12). The ansatz for the BPS monopole solution (2.9) was the covariance under the rotation of the diagonal $S O(3)$ subgroup of $S O(3)_{\text {gauge }} \times$ $S O(3)_{\text {space. }}$ In order to solve eq. (2.12), we put the following ansatz for the $U(1)$ fields $\left(a_{i}^{0}, \varphi^{0}\right)$ respecting the covariance under the generalized rotation, in which we rotate also the parameter $\theta_{i j}:^{\dagger}$

$$
a_{i}^{0}=\theta_{i j} x_{j} A(r), \quad \varphi^{0}=\theta_{i j} \epsilon_{i j k} x_{k} B(r),
$$

where $A(r)$ and $B(r)$ are functions of $r$ to be determined. Putting these ansatz (2.13) and the explicit forms of the BPS solution (2.9) into the differential equation (2.12), we obtain the following two equations as the coefficients of different tensor structures:

$$
\begin{aligned}
& -A+B+r B^{\prime}+\frac{1}{4 r^{2}} W(W+2 F)=0 \\
& A^{\prime}+2 B^{\prime}-\frac{d}{d r}\left[\frac{1}{4 r^{2}} W(W+2 F)\right]=0 .
\end{aligned}
$$

The solution to eqs. (2.14) and (2.15) is given by

$$
A(r)=\frac{1}{4 r^{2}} W(W+2 F)-2 \frac{c_{1}}{r^{3}}+c_{2}, \quad B(r)=\frac{c_{1}}{r^{3}}+c_{2},
$$

with two arbitrary constant parameters, $c_{1}$ and $c_{2}$. The parts in (2.16) containing these constant parameters are actually solution to the homogeneous part of eq. (2.12):

$$
\partial_{i} \varphi^{0}+\epsilon_{i j k} \partial_{j} a_{k}^{0}=0 .
$$

Since the $c_{2}$ part of the scalar $\varphi^{0}$ diverges at the infinity, we put $c_{2}=0$. As for the $c_{1}$ part, a careful substitution into the left hand side of eq. (2.17) gives in fact a term proportional to $\partial_{i} \partial_{i}(1 / r)=-4 \pi \delta^{3}(\boldsymbol{r})$. Hence the $c_{1}$ part is not a solution at the origin, and we shall also put $c_{1}=0$. Finally the desired solution of the equation (2.12) is

$$
a_{i}^{0}=\theta_{i j} x_{j} \frac{1}{4 r^{2}} W(W+2 F), \quad \varphi^{0}=0 .
$$

Note in particular that the scalar field receives no correction to this order. Since the whole solution has the same leading asymptotic behavior as the BPS solution (2.9), we find that the non-commutativity does not change the mass of the monopole.

\footnotetext{
${ }^{\dagger}$ The generalized rotational covariance for $a_{i}^{0}$ allows two other terms with different structures, $\epsilon_{i j k} \theta_{j k}$ and $x_{i} \epsilon_{j k l} \theta_{j k} x_{l}$. However, eq. (2.12) implies the vanishing of these two terms.
} 


\section{Non-commutative eigenvalue equation}

The configuration of the D-string suspended between two parallel D3-branes is described by the deformation of the surface of the D3-branes in the spirit of the BIon (Born-Infeld soliton) physics $[14,15]$. The extent of this deformation of the D3-brane surface is given by the eigenvalues of the scalar field on the D3-branes. We saw in the previous section that there is no additional contribution of $O(\theta)$ in the scalar field configuration. However, since we are now dealing with the theory with the $*$-product, the eigenvalue problem should be different from that in the usual commutative case. In this section, we see that the $O(\theta)$ terms are actually generated in the eigenvalues of the scalar field.

We propose that the eigenvalue equation for a hermitian matrix valued function $M$ to be considered in the non-commutative case is

$$
M * \boldsymbol{v}=\lambda * \boldsymbol{v}
$$

where $\boldsymbol{v}$ and $\lambda$ are the eigenvector and the eigenvalue, respectively. Though there are other candidates for the non-commutative eigenvalue equation, the present one (3.1) has advantages over the others in various respects as we shall see in this and the final sections.

For solving (3.1) to $O(\theta)$, let us make the expansion

$$
M=M_{0}+M_{1}, \quad \boldsymbol{v}=\boldsymbol{v}_{0}+\boldsymbol{v}_{1}, \quad \lambda=\lambda_{0}+\lambda_{1},
$$

where the subscript number specifies the order of $\theta$. Then, the $O\left(\theta^{0}\right)$ part of $(3.1)$ is $M_{0} \boldsymbol{v}_{0}=$ $\lambda_{0} \boldsymbol{v}_{0}$, and the $O(\theta)$ part reads

$$
M_{0} \boldsymbol{v}_{1}+M_{1} \boldsymbol{v}_{0}+\frac{i}{2}\left\{M_{0}, \boldsymbol{v}_{0}\right\}=\lambda_{0} \boldsymbol{v}_{1}+\lambda_{1} \boldsymbol{v}_{0}+\frac{i}{2}\left\{\lambda_{0}, \boldsymbol{v}_{0}\right\}
$$

Multiplying $\boldsymbol{v}_{0}^{\dagger}$ from the left, we obtain the formula which gives the $O(\theta)$ part of the eigenvalue:

$$
\lambda_{1}=\frac{1}{\boldsymbol{v}_{0}^{\dagger} \boldsymbol{v}_{0}}\left(\frac{i}{2} \boldsymbol{v}_{0}^{\dagger}\left\{M_{0}-\lambda_{0} \mathbb{I}, \boldsymbol{v}_{0}\right\}+\boldsymbol{v}_{0}^{\dagger} M_{1} \boldsymbol{v}_{0}\right) .
$$

In view of the application to the present NCBPS solution, let us consider the particular case with

$$
M_{0}=m_{0}(\boldsymbol{r}) \widehat{x}_{a} \sigma_{a} \quad\left(\widehat{x}_{i} \equiv x_{i} / r\right), \quad M_{1}=0
$$

and hence $\lambda_{0}= \pm m_{0}(\boldsymbol{r})$ and $\widehat{x}_{a} \sigma_{a} \boldsymbol{v}_{0}= \pm \boldsymbol{v}_{0}$. Then, eq. (3.4) is calculated to give

$$
\lambda_{1}=\frac{i}{2 \boldsymbol{v}_{0}^{\dagger} \boldsymbol{v}_{0}} \frac{m_{0}(\boldsymbol{r})}{r} \theta_{i j}\left(\boldsymbol{v}_{0}^{\dagger} \sigma_{i} \partial_{j} \boldsymbol{v}_{0} \mp \widehat{x}_{i} \boldsymbol{v}_{0}^{\dagger} \partial_{j} \boldsymbol{v}_{0}\right)=-\frac{m_{0}(\boldsymbol{r})}{2 r^{2}} \theta_{i} \widehat{x}_{i}
$$


with $\theta_{i} \equiv(1 / 2) \epsilon_{i j k} \theta_{j k}$. Note that $\lambda_{1}$ (3.6) is independent of the sign of $\lambda_{0}$. We obtained the last expression of (3.6) using the explicit form $\boldsymbol{v}_{0}=\left(x_{1}-i x_{2}, \pm r-x_{3}\right)^{\mathrm{T}}$. However, the general formula for $\lambda_{1}$, eq. (3.4), is in fact invariant under the local phase and scale transformation of $\boldsymbol{v}_{0}$ due to the identity $\boldsymbol{v}_{0}^{\dagger}\left\{M_{0}-\lambda_{0} \mathbb{I}, f \mathbb{I}\right\} \boldsymbol{v}_{0}=0$ valid for any $f(\boldsymbol{r})$. This corresponds to the fact that the eigenvalue $\lambda$ of (3.1) is invariant under the right multiplication $\boldsymbol{v} \rightarrow \boldsymbol{v} * h$ for an arbitrary $h(\boldsymbol{r})$. We shall discuss the gauge transformation property of the eigenvalues in the final section.

Let us evaluate various eigenvalues of the system using the formula (3.6). First, the scalar eigenvalues are obtained by substituting $m_{0}(\boldsymbol{r})=F(r) / 2$ as

$$
\lambda_{\Phi}= \pm \frac{1}{2} F(r)-\frac{\theta_{i} \widehat{x}_{i}}{4 r^{2}} F(r)+O\left(\theta^{2}\right) .
$$

Next, we shall consider the eigenvalues of the magnetic field $B_{i} \equiv(1 / 2) \epsilon_{i j k} F_{j k}$ near the infinity $r \rightarrow \infty$. The magnetic field itself is given from the solution (2.18) as ${ }^{\ddagger}$

$$
B_{i}=-\frac{\widehat{x}_{i}}{2 r^{2}} \widehat{x}_{a} \sigma_{a}+\frac{C}{2 r^{3}}\left(\delta_{i j}-3 \widehat{x}_{i} \widehat{x}_{j}\right) \theta_{j} \mathbb{I}+O\left(\frac{1}{r^{4}}\right) .
$$

We would like to evaluate the $O(\theta)$ contribution to the eigenvalues by putting $m_{0}(\boldsymbol{r})=$ $-\widehat{x}_{i} / 2 r^{2}$ and $M_{1}(\boldsymbol{r}, \theta)=C\left(\delta_{i j}-3 \widehat{x}_{i} \widehat{x}_{j}\right) \theta_{j} \mathbb{1} / 2 r^{3}$. Since $m_{0}(\boldsymbol{r})$ in this case is $O\left(1 / r^{2}\right)$, using the formula (3.6), the order of the correction to the eigenvalues from this part is found as $O\left(1 / r^{4}\right)$. Thus near the infinity, the $O\left(1 / r^{3}\right)$ part of the eigenvalues of the magnetic field is saturated by $m_{1}$.

\section{Interpretation of the eigenvalues}

In this section, we shall see how the eigenvalues (3.7) and (3.8) reproduce the brane configuration depicted in fig. 1. In the brane picture, the end of the D-string is seen as a magnetic charge in a single D3-brane worldvolume theory. The prediction from the brane configuration is that the magnetic charge on each end of the D-string is actually moved in different directions between the upper and lower D3-branes, as shown in fig. 1. This shift is specified by the spatial vector $\delta_{i}$.

Now, the eigenvalues of the magnetic field (3.8) indicate that the $U(1)$ part of the magnetic field exhibits a dipole structure. This structure is exactly the one expected from the brane picture above. Noting that the zero-th order solution (2.9) represents $-1 / 2$ charge on

\footnotetext{
${ }_{\ddagger}^{\ddagger}$ Since the definition of the magnetic field contains the $*$-product in itself, we should calculate also the Poisson bracket term. However, this term contributes only to the $O\left(1 / r^{4}\right)$ part in $(3.8)$.
} 
the upper D3-brane and 1/2 charge on the lower, it is easy to derive the non-locality $\delta_{i}$ as

$$
\delta_{i}=C \theta_{i} .
$$

This result verifies the prediction of ref. [11] with the identification $C=U$.

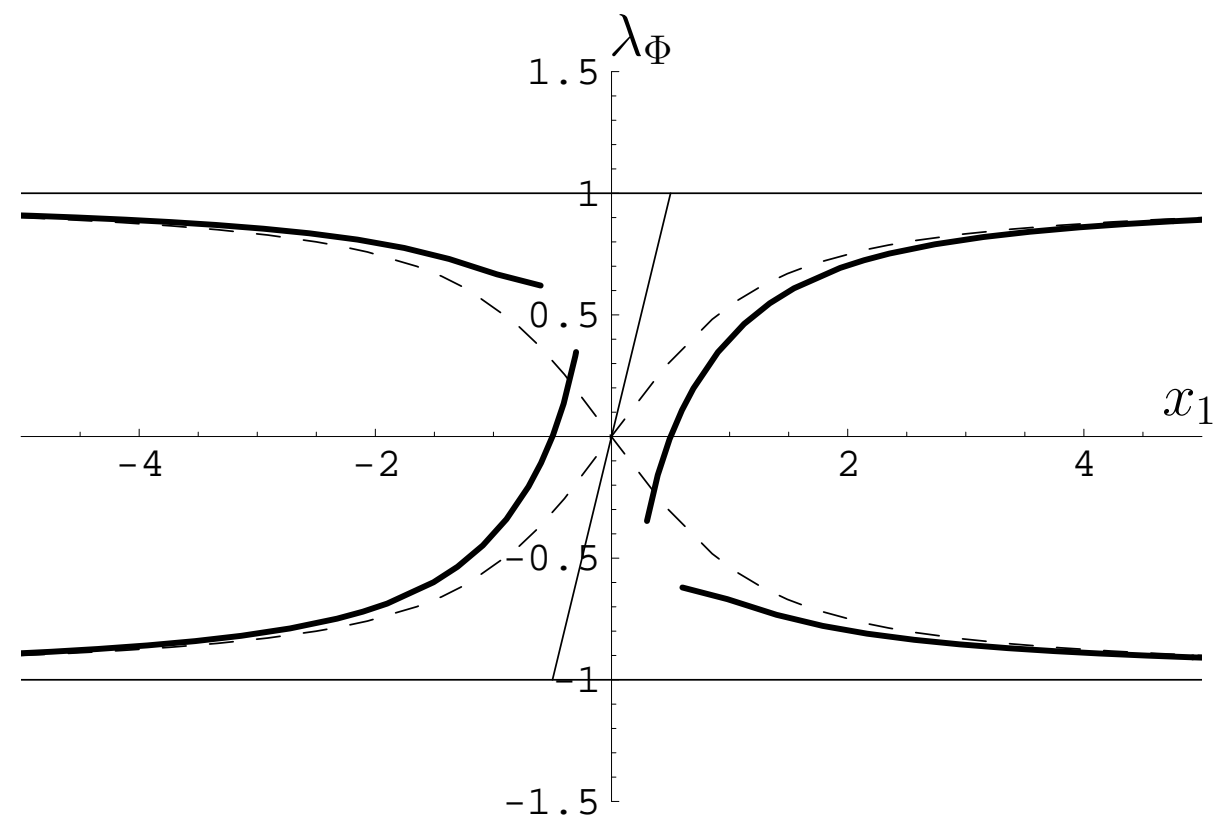

Figure 2: The eigenvalues of the solution for the scalar field. We choose $C=2$ and $\theta_{23}=1 / 2$, and other $\theta$ 's are set to zero.

Although the magnetic charges are expected to indicate only the locations of the ends of the D-string, the eigenvalues of the scalar field must reproduce not only the locations of the ends but also the slope of the D-string. In fact, the asymptotic behavior of the eigenvalues (3.7) is given using (2.11) as

$$
\begin{aligned}
\lambda_{\Phi} & = \pm \frac{C}{2} \mp \frac{1}{2 r}+\left(-\frac{C}{4 r^{2}}+\frac{1}{4 r^{3}}\right) \theta_{i} \widehat{x}_{i}+O\left(e^{-C r}\right) \\
& = \pm \frac{C}{2} \mp \frac{1}{2}\left|x_{i} \mp\left(\frac{C}{2}-\frac{1}{2 r}\right) \theta_{i}\right|^{-1}+O\left(e^{-C r}\right) .
\end{aligned}
$$

Eq. (4.2) implies first that in the upper and the lower D3-brane the end of the D-string sits at $x_{i}=C \theta_{i} / 2$ and $x_{i}=-C \theta_{i} / 2$, respectively. Hence the non-locality is precisely given by $\delta_{i}=C \theta_{i}$, in agreement with the result (4.1) from the magnetic field. Secondly, in order to read off the slope of the D-string from (4.2), we rewrite it as

$$
\lambda_{\Phi}= \pm \frac{C}{2} \mp \frac{1}{2}\left|x_{i}-\lambda_{\Phi} \theta_{i}\right|^{-1}+O\left(e^{-C r}\right) .
$$


This equation means that, for a given value of $\lambda_{\Phi}$, the corresponding worldvolume coordinate is located on a sphere with its center at $x_{i}=\lambda_{\Phi} \theta_{i}$. Interpreting the trajectory of the center as the D-string, our analysis reproduces precisely the tilt of the suspended D-string.

In fig. 2 we present the curves of the eigenvalues of the scalar field. The thin straight lines represent the brane configuration of fig. 1. The dashed curves denote the eigenvalues of the scalar field with $\theta=0$. Comparing these with the bold curves representing the eigenvalues (3.7) with $\theta \neq 0$, we can read off the simple brane configuration of ref. [11]. (The reason why the bold curves are cutoff for small $\left|x_{1}\right|$ in fig. 2 is that the $O(\theta)$ term of the scalar eigenvalues (3.7) are actually divergent at the origin $r=0$. We shall discuss this problem in the next section.)

\section{Summary and discussion}

In this paper, we solved the BPS equation of the NCSYM to the first non-trivial order in $\theta$. Evaluating the eigenvalues of the solution, we explicitly showed that the solution exhibits the brane configuration of the tilted D-string, given in ref. [11]. Magnetic field has the dipole structure, and the scalar field is also shifted to reproduce the tilted trajectory of the D-string.

Some comments are in order. Our first comment is on the gauge transformation property of the eigenvalue $\lambda$ in our non-commutative eigenvalue equation (3.1). Of course, the eigenvalue $\lambda$ in eq. (3.1) is never strictly invariant under the local gauge transformation of $M$

$$
M \rightarrow U^{-1} * M * U
$$

where $U^{-1}$ is the inverse of $U$ with respect to the $*$-product, $U * U^{-1}=U^{-1} * U=\mathbb{I}$. However, we can show that the eigenvalue has a fairly nice property under (5.1). Consider an infinitesimal gauge transformation $\delta_{\epsilon}$ on $M$ with $U=\mathbb{I}+i \epsilon\left(\epsilon^{\dagger}=\epsilon\right)$,

$$
\delta_{\epsilon} M=i(M * \epsilon-\epsilon * M) .
$$

Letting $\delta_{\epsilon}$ act on (3.1) and $*$-multiplying the resultant equation by $\boldsymbol{v}^{\dagger}$ from the left, we obtain

$$
\boldsymbol{v}^{\dagger} * \delta_{\epsilon} \lambda * \boldsymbol{v}=i \boldsymbol{v}^{\dagger} *(\lambda * \epsilon-\epsilon * \lambda) * \boldsymbol{v}
$$

Taking the $O(\theta)$ part of (5.3) and using $\delta_{\epsilon} \lambda_{0}=0, \delta_{\epsilon} \lambda_{1}$ is given as

$$
\delta_{\epsilon} \lambda_{1}=\boldsymbol{v}_{0}^{\dagger}\left\{\epsilon, \lambda_{0}\right\} \boldsymbol{v}_{0},
$$

for a normalized $\boldsymbol{v}_{0}$. Eq. (5.4) implies that, at least to $O(\theta)$, the gauge transformation corresponds to a coordinate transformation on the eigenvalue $\lambda(\boldsymbol{r})$. In the $U(2)$ case with $\epsilon(\boldsymbol{r})=$ $\epsilon_{a}(\boldsymbol{r}) \sigma_{a}+\epsilon_{0}(\boldsymbol{r}) \mathbb{I}$, the form of the coordinate transformation is $\delta_{\epsilon} x_{i}=-\theta_{i j}\left(\partial_{j} \epsilon_{0}+\boldsymbol{v}_{0}^{\dagger} \sigma_{a} \boldsymbol{v}_{0} \partial_{j} \epsilon_{a}\right)$. 
Therefore, we have shown that the eigenvalue of (3.1) for $M$ and the one for $U^{-1} * M * U$ are related by a coordinate transformation on the D3-branes and hence are physically equivalent, at least to the first non-trivial order in $\theta$. Recalling also that the eigenvalue of (3.1) is independent of the choice of the eigenvector and that the scalar eigenvalue (3.7) has an invariance under the simultaneous rotation of $x_{i}$ and $\theta_{i j}$, the eigenvalue equation (3.1) we have adopted is a satisfactory one. These good properties, in particular, the gauge transformation property of $\lambda$, are expected to persist to higher orders in $\theta$.

Our second comment is on another type of non-commutative eigenvalue equation,

$$
M * \boldsymbol{v}=\boldsymbol{v} * \lambda
$$

where, compared with (3.1), $\boldsymbol{v}$ and $\lambda$ are interchanged on the right hand side. Eq. (5.5) has a property that the eigenvalue $\lambda$ is invariant under the gauge transformation (5.1) of $M$. However, for a given $M$ the eigenvalues are not unique and do depend on the choice of the eigenvectors $\boldsymbol{v}$. What is worse, in the analysis similar to those in Sec. 3 by adopting (5.5), we can show that it is impossible to obtain the the scalar eigenvalues possessing an invariance under the simultaneous rotation of $x_{i}$ and $\theta_{i j}$. These are the reasons why we did not adopt $(5.5)$.

Our final comment is concerning the singular nature of the scalar eigenvalues (3.7) at the origin $r=0$. Since we have adopted the eigenvalue equation (3.1), the matrix whose diagonal entries are these eigenvalues is no longer a solution of the BPS equation. Hence although the eigenvalues (4.2) diverge at the origin, the energy of the configuration is still finite. We would need a technique beyond the expansion in powers of $\theta$ to dissolve the singularity at the origin.

Our analysis in this paper can straightforwardly be applied to the case of dyons. We obtain a result consistent with the non-locality predicted by [11]. It would be an interesting subject to study the non-commutative 1/4 BPS dyons using our formulation.

\section{Note added}

While writing this paper, we became aware of the paper [16] which has an overlap concerning the solution of Sec. 2 .

\section{Acknowledgments}

We would like to thank A. Hashimoto, T. Hirayama, S. Imai, H. Kawai, I. Kishimoto, T. Kugo, Y. Okawa and S. Terashima for valuable discussions and comments. This work 
is supported in part by Grant-in-Aid for Scientific Research from Ministry of Education, Science, Sports and Culture of Japan (\#3160, \#09640346, \#4633). The work of K. H. and S. M. is supported in part by the Japan Society for the Promotion of Science under the Predoctoral Research Program.

\section{References}

[1] J. H. Schwarz, "Lectures on superstring and M theory dualities", Nucl. Phys. Proc. Suppl. 55B (1997) 1, hep-th/9607201.

[2] K. Dasgupta and S. Mukhi, "BPS nature of 3-string junctions", Phys. Lett. B423 (1998) 261, hep-th/9711094.

[3] O. Bergman, "Three-pronged strings and 1/4 BPS states in $\mathrm{N}=4$ super Yang-Mills theory", Nucl. Phys. B525 (1998) 104, hep-th/9712211.

[4] K. Hashimoto, H. Hata, and N. Sasakura, "Three-string junction and BPS saturated solutions in SU(3) supersymmetric Yang-Mills theory", Phys. Lett. B431 (1998) 303, hep-th/9803127.

[5] K. Hashimoto, H. Hata, and N. Sasakura, "Multipronged strings and BPS saturated solutions in SU(N) supersymmetric Yang-Mills theory", Nucl. Phys. B535 (1998) 83, hep-th/9804164.

[6] T. Kawano and K. Okuyama, "String network and 1/4 BPS states in N=4 SU(N) supersymmetric Yang-Mills theory", Phys. Lett. B432 (1998) 338, hep-th/9804139.

[7] K. Lee and P. Yi, "Dyons in $\mathrm{N}=4$ supersymmetric theories and three-pronged strings", Phys. Rev. D58 (1998) 066005, hep-th/9804174.

[8] A. Connes, M. R. Douglas, and A. Schwarz, "Noncommutative geometry and matrix theory: Compactification on tori", JHEP 02 (1998) 003, hep-th/9711162.

[9] M. R. Douglas and C. Hull, "D-branes and the noncommutative torus", JHEP 02 (1998) 008, hep-th/9711165.

[10] N. Seiberg and E. Witten, "String theory and noncommutative geometry", JHEP 09 (1999) 032, hep-th/9908142.

[11] A. Hashimoto and K. Hashimoto, "Monopoles and dyons in non-commutative geometry", hep-th/9909202. 
[12] E. B. Bogomol'nyi, "The stability of classical solutions", Sov. J. Nucl. Phys. 24 (1976) 449.

[13] M. K. Prasad and C. M. Sommerfield, "An exact classical solution for the 't Hooft monopole and the Julia-Zee dyon", Phys. Rev. Lett. 35 (1975) 760.

[14] C. G. Callan Jr. and J. M. Maldacena, "Brane dynamics from the Born-Infeld action", Nucl. Phys. B513 (1998) 198, hep-th/9708147.

[15] A. Hashimoto, "The shape of branes pulled by strings," Phys. Rev. D57 (1998) 6441, hep-th/9711097.

[16] D. Bak, "Deformed Nahm equation and a noncommutative BPS monopole," hep-th/9910135. 